\title{
Thyroid function alters during neoadjuvant chemotherapy in breast cancer patients: results from the NEOZOTAC trial (BOOG 2010-01)
}

\author{
S. de Groot • L. G. M. Janssen • A. Charehbili • E. M. Dijkgraaf • \\ V. T. H. B. M. Smit - L. W. Kessels • A. van Bochove - H. W. M. van Laarhoven • \\ E. Meershoek-Klein Kranenbarg • A. E. van Leeuwen-Stok • C. J. H. van de Velde \\ H. Putter · J. W. R. Nortier · J. J. M. van der Hoeven · H. Pijl • J. R. Kroep
}

Received: 10 October 2014/ Accepted: 21 December 2014/Published online: 4 January 2015

(C) The Author(s) 2015. This article is published with open access at Springerlink.com

\begin{abstract}
This side study investigated the effect of chemotherapy on thyroid function and the extent to which it can predict pathological complete response (pCR) in patients with early breast cancer taking part in NEOZOTAC phase III trial, randomizing between neoadjuvant chemotherapy with or without additional zoledronic acid. Moreover, we examined the impact of thyroid function on toxicity. Serum samples of 38 patients were available for analyses. Free thyroxin (fT4) and thyroid stimulating hormone (TSH) levels were compared between baseline and before the 6th cycle and between subjects with and without $\mathrm{pCR}$. The relation between toxicity and the variation in fT4 and TSH levels during chemotherapy was tested. Samples at baseline and before the 6th cycle were available for 31 and 21 patients, respectively. The mean baseline fT4 level was $16.0 \mathrm{pmol} / \mathrm{L}$ and TSH level $1.11 \mathrm{mU} / \mathrm{L}$, and these did
\end{abstract}

S. de Groot · A. Charehbili · E. M. Dijkgraaf .

J. W. R. Nortier · J. J. M. van der Hoeven · J. R. Kroep ( $\square)$ Department of Clinical Oncology, Leiden University Medical Center, Abinusdreef 2, P.O. Box 9600, 2300 RC Leiden,

The Netherlands

e-mail: j.r.kroep@lumc.nl

S. de Groot

e-mail: s.de_groot2@lumc.nl

L. G. M. Janssen · H. Pijl

Department of Endocrinology, Leiden University Medical

Center, Leiden, The Netherlands

A. Charehbili · E. Meershoek-Klein Kranenbarg ·

C. J. H. van de Velde

Department of Surgery, Leiden University Medical Center,

Leiden, The Netherlands

V. T. H. B. M. Smit

Department of Pathology, Leiden University Medical Center,

Leiden, The Netherlands not differ between both arms at each time point. During six cycles of chemotherapy, fT4 levels decreased $(p=0.0001)$, and TSH levels increased significantly $(p=0.019)$. Interestingly, the decrease of fT4 was significantly greater in patients without nausea, vomiting, or neuropathy, than in patients with those side effects ( $p=0.037, p=0.043$, and $p=0.050$, respectively). Baseline TSH levels tended to be higher in patients with $\operatorname{pCR}(p=0.035$ univariate analysis and $p=0.074$ multivariate analysis). Chemotherapy blunts thyroid function, which was associated with less side effects. These data urge further evaluation of the effects of thyroid function on toxicity and outcome of breast cancer therapy.

Keywords Breast cancer - Thyroid hormones . Chemotherapy $\cdot$ Toxicity $\cdot \mathrm{pCR}$

L. W. Kessels

Department of Oncology, Deventer Ziekenhuis, Deventer, The Netherlands

A. van Bochove

Department of Medicine, Zaans Medisch Center, Zaandam, The Netherlands

H. W. M. van Laarhoven

Department of Medical Oncology, Academisch Medisch

Centrum, Amsterdam, The Netherlands

A. E. van Leeuwen-Stok

Dutch Breast Cancer Research Group (BOOG), Amsterdam, The Netherlands

H. Putter

Department of Medical Statistics, Leiden University Medical Center, Leiden, The Netherlands 


\section{Introduction}

Several epidemiological studies show a positive association between plasma thyroid hormones (TH) - triiodothyronine $\left(\mathrm{T}_{3}\right)$ and the prohormone thyroxine $\left(\mathrm{T}_{4}\right)$-levels and breast cancer risk [1-3], which supports the notion that $\mathrm{TH}$ promote tumor growth [4]. Indeed, in patients with hypothyroidism, breast cancer tends to be diagnosed at an older age and in a less advanced stage, suggesting that hypothyroidism protects against breast cancer and is associated with more indolent disease [5]. Hypothyroidism may protect against breast cancer, because $\mathrm{TH}$ directly stimulate tumor growth via $\mathrm{T}_{3}$ receptors (TR) [5], or because hypothyroidism is associated with reduced serum concentrations of insulin-like growth factor 1 [4, 6, 7]. Moreover, TH have estrogen-like effects [8,9], whereby $\mathrm{T}_{3}$ may have the ability to mimic or enhance the effects of estradiol on breast cancer proliferation by binding to the estrogen receptor [9, 10]. In rodent tumor models, $\mathrm{TH}$ stimulate tumor growth, metastasis, and angiogenesis via integrin $\alpha v \beta 3$, possibly mediated by phosphatidylinositol-3-kinase and MAPK $[11,12]$. Clinical studies show that treatmentinduced hypothyroidism, either by concomitant propylthiouracil or by cancer treatment itself, is associated with a favorable outcome in several cancer types $[6,13]$. In a case control study, euthyroid patients with breast cancer had significantly higher TH levels than their healthy controls [14]. Thus, although only a few cases of overt hypothyroidism (probably induced by locoregional radiotherapy) after breast cancer therapy were reported [15-17], even a slight decrease of THs in breast cancer patients treated with chemotherapy [18] could be of clinical relevance.

The influence of TH on effectiveness of chemotherapy remains unclear to date [19]. We aimed to elucidate the effect of chemotherapy on thyroid function and the predictive value of thyroid function on pathological complete response (pCR) in early breast cancer patients receiving neoadjuvant chemotherapy.

\section{Patients and methods}

Patients

Women eligible for the NEOZOTAC study had a histologically confirmed HER2-negative stage II or III breast cancer, and further in- and exclusion criteria have been described previous [20]. A subgroup participated in a side study measuring plasma fT4 and TSH concentrations before cycle 1, 2, and 6 of chemotherapy. Eleven of the participating centers of the NEOZOTAC participated in the side study; patients gave informed consent for extra blood sampling.
Study design

The NEOZOTAC study was an open label randomized phase III study, running in 26 hospitals in the Netherlands, which compared the efficacy of neoadjuvant chemotherapy with or without zoledronic acid. Eleven of the 26 hospitals participated in the side study. The study was conducted in accordance with the Declaration of Helsinki and approved by the Ethics Committees of the participating hospitals in agreement with the Dutch law for medical research involving humans.

\section{Drugs}

Women received TAC $\left(75 \mathrm{mg} / \mathrm{m}^{2}\right.$ of docetaxel i.v. in $1 \mathrm{~h}$, $50 \mathrm{mg} / \mathrm{m}^{2}$ of doxorubicin i.v. in $15 \mathrm{~min}$ and $500 \mathrm{mg} / \mathrm{m}^{2}$ of cyclophosphamide i.v. in $1 \mathrm{~h}$ ) on the first day of each of six 21-day cycles with or without $4 \mathrm{mg}$ zoledronic acid i.v. in 15 min within $24 \mathrm{~h}$ after infusion of chemotherapy. Patients received premedication with dexamethasone $(8 \mathrm{mg}$, b.i.d. the day before, the day of and the day after chemotherapy administration) in order to prevent fluid retention and hypersensitivity reactions. Granulocyte-colony stimulating factor (pegfilgrastim $6 \mathrm{mg}$ ) support was given the day after chemotherapy administration.

Blood sampling and analysis

Non-fasting blood samples were obtained directly before chemotherapy administration (baseline), before the second and before the sixth chemotherapy cycle (end of therapy) to measure fT4 and TSH levels. Samples were collected in EDTA tubes and directly kept on ice after drawing. After centrifuging, the supernatant was stored at $-80{ }^{\circ} \mathrm{C}$, and at the end of the study sent to the Leiden University Medical Center (LUMC) for analysis.

TSH and fT4 were determined by Modular Analytics E170 (Roche Diagnostics, Almere, The Netherlands) with chemiluminescence methods by the clinical laboratory of the LUMC. The following reference ranges were used; TSH $0.3-4.8 \mathrm{mU} / \mathrm{L}$ and $\mathrm{fT} 4$ is $12-22 \mathrm{pmol} / \mathrm{L}$.

Response and toxicity evaluation

Central review of operation specimens was performed according to Dutch guidelines. Tumor regression was scored according to the Miller and Payne classification [21]. Pathological complete response ( $\mathrm{pCR}$ ) was defined as the absence of residual invasive cancer within the breast and lymph nodes.

All adverse events, grade II or more (according to NCI-CTCv4.0) reported spontaneously by the patient or observed by the treating physician or investigator, were recorded on an adverse event case report form. The relationship to the study $\operatorname{drug}(\mathrm{s})$ was recorded. 
Statistical analysis

We performed an intention-to-treat analysis. All parameters were tested for normality using the KolmogorovSmirnov test, with Bonferroni adjustment when evaluated in subgroups. The normally distributed parameters were summarized as 'mean \pm standard error (SE)' and compared using t-tests for paired groups. All $t$-tests and regression models were 2 -sided with a significance level of 0.05. Univariate and multivariate odds ratios (OR), $95 \%$ confidence intervals (95\% CI), and P values were calculated by applying logistic regression analysis. All data were analyzed using Statistical Package for Social Sciences (SPSS) software ${ }^{\mathrm{TM}} 20.0$ (IBM Corp., Armonk, NY, USA).

\section{Results}

Patient characteristics

Serum samples of 38 of the 105 patients who participated in the side study measuring fT4 and TSH concentrations at different time-points during chemotherapy treatment were of sufficient quality and quantity for analysis of fT4 and TSH. None of the patients had a thyroid disorder or were on thyroid replacement therapy at baseline, and further clinical characteristics of the 38 women are given in Table 1. Patient characteristics are comparable with the characteristics of the whole cohort of the NEOZOTAC trial [20].

Thyroid function in breast cancer patients during chemotherapy

Serum samples at baseline, before the 2nd chemotherapy cycle and at end of treatment were available for 31, 30, and 21 patients, respectively. Baseline fT4 and TSH levels were compared with values before the 2 nd and 6th chemotherapy cycle. FT4 levels decreased, and TSH levels increased significantly during chemotherapy treatment. None of the patients had $\mathrm{TH}$ values under or above the reference value. Mean values of fT4 and TSH before cycle 1, 2, and 6 are shown in Fig. 1. The given $P$ values are based on the tested values of the first and the last cycles. There were no significant differences between subjects solely treated with TAC chemotherapy and subjects treated with zoledronic acid as an adjunct to TAC with respect to the mean $\mathrm{FT} 4$ and TSH levels at each time point. Chemotherapy adjustments were made in four patients; one patient stopped after 4 cycles TAC, one patients after 5 cycles, and two patients received only less cycles of docetaxel ( 1 and 3 cycles less, respectively), because of toxicity. No dose reductions were done. No significant differences were seen in the decline of fT4 $(P=0.354)$ and increase of TSH $(P=0.770)$ between patients with
Table 1 Patient characteristics

Patients $(N=38)$ NEOZOTAC

Median Age (range), Years

$49(34-65)$

WHO-status

0

$34(89.5 \%)$

1

Unknown

$2(5.8 \%)$

$2(5.8 \%)$

T-classification

cT2

$22(57.9 \%)$

cT3 or cT4

$16(42.1 \%)$

$\mathrm{N}$-classification

cNO

$19(50.0 \%)$

$\mathrm{cN}+$

$19(50.0 \%)$

Tumor type

Ductal

$27(71.1 \%)$

Lobular

$7(18.4 \%)$

Other

$3(7.9 \%)$

Unknown

$1(2.6 \%)$

Hormone receptor status

$\mathrm{ER}+$ and/or PR+

$33(86.8 \%)$

ER - and PR-

$5(13.2 \%)$

Allocated treatment

TAC

$20(52.6 \%)$

$\mathrm{TAC}+\mathrm{ZA}$

$18(47.4 \%)$

pCR

Yes

$6(15.8 \%)$

No

$31(81.6 \%)$

Unknown

$1(2.6 \%)$

$E R$ estrogen receptor, $P R$ progesterone receptor, $Z A$ zoledronic acid, $p C R$ pathologic complete response

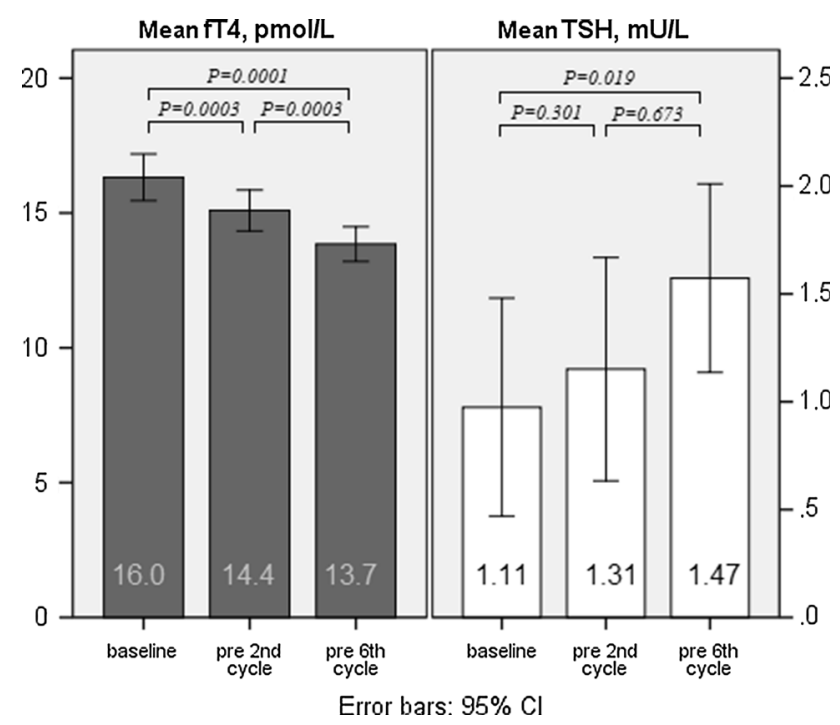

Fig. 1 Serum fT4 and TSH levels baseline and during chemotherapy 
chemotherapy adjustments compared with patients who received all six cycles of TAC.

Association between alterations of thyroid function and side effects during chemotherapy

The relation between toxicity, per side effect of CTC grade II or more, and baseline values, as well as the variation in fT4 and TSH levels during chemotherapy was tested. There was no significant association between baseline fT4 and TSH and manifest side effects. However, a small decline of fT4 in the course of treatment, as reflected by a high ratio of fT4 levels at cycle 6 over fT4 levels at baseline, was associated with neuropathy, nausea, and vomiting grade II or more (Table 2). The ratio of TSH levels between pre and post-chemotherapy was not associated with side effects.

The predictive value of thyroid function on efficacy of chemotherapy

FT4 and TSH levels were compared between subjects with and without pCR. PCR was achieved in $16 \%$ of the patients (6/37 and 1 unknown), and the pCR rate varied in the different subtypes, which is comparable with the whole cohort of the NEOZOTAC trial [20]. In hormone receptorpositive breast cancer, the pCR rate was $12.5 \%(4 / 32)$, compared to $40 \%(2 / 5)$ in the triple-negative patients.

FT4 and TSH levels were not related to pCR (Table 3). High TSH levels were associated with pCR in univariate analysis, but this was not significant in multivariate analysis. Although axillary lymph node status, tumor size, and hormone receptor status were not significantly associated with pCR, these clinicopathological factors were included in the multivariate model, as they were associated with pCR in earlier research $[22,23]$. The total dose of chemotherapy was also included in the multivariate model.

\section{Discussion}

This study shows that thyroid function declines during treatment in stage II/III HER2-negative breast cancer patients treated with neoadjuvant TAC chemotherapy. Interestingly, fT4 levels decreased less in patients with side effects of therapy compared to patients without side effects.

The decline of fT4 concentrations and increase of TSH concentrations during TAC observed in our study may reflect damage to the thyroid gland inflicted by the

Table 2 Ratio of fT4 levels at cycle 6 over fT4 levels at baseline in relation to experienced side effects

\begin{tabular}{|c|c|c|c|c|c|c|c|}
\hline Side effect (grade II or more) & $N$ & Ratio fT4 (\%) & $\mathrm{OR}^{\mathrm{a}}(95 \% \mathrm{CI})$ & $P$ value & Ratio TSH (\%) & $\mathrm{OR}^{\mathrm{a}}(95 \% \mathrm{CI})$ & $P$ value \\
\hline \multirow[t]{2}{*}{ Neuropathy } & Yes 18 & 89 & $2.56(1.00-6.56)$ & 0.050 & 200 & $1.02(0.97-1.07)$ & 0.517 \\
\hline & No 12 & 81 & & & 163 & & \\
\hline \multirow[t]{2}{*}{ Vomiting } & Yes 7 & 95 & $3.36(1.04-10.87)$ & 0.043 & 163 & $0.99(0.93-1.05)$ & 0.664 \\
\hline & No 23 & 84 & & & 191 & & \\
\hline \multirow[t]{2}{*}{ Nausea } & Yes 20 & 90 & $2.99(1.07-8.37)$ & 0.037 & 150 & $0.96(0.91-1.01)$ & 0.094 \\
\hline & No 10 & 80 & & & 255 & & \\
\hline \multirow[t]{2}{*}{ Alopecia } & Yes 13 & 91 & $2.45(0.98-6.13)$ & 0.056 & 171 & $0.99(0.94-1.04)$ & 0.663 \\
\hline & No 17 & 83 & & & 196 & & \\
\hline \multirow[t]{2}{*}{ Fatigue } & Yes 27 & 86 & $1.17(0.38-3.61)$ & 0.780 & 185 & $1.00(0.93-1.08)$ & 0.996 \\
\hline & No 3 & 84 & & & 185 & & \\
\hline \multirow[t]{2}{*}{ Mucositis } & Yes 10 & 87 & $1.11(0.56-2.22)$ & 0.759 & 222 & $1.02(0.98-1.07)$ & 0.352 \\
\hline & No 20 & 86 & & & 166 & & \\
\hline \multirow[t]{2}{*}{ Diarrhea } & Yes 7 & 93 & $2.38(0.91-6.22)$ & 0.078 & 107 & $0.92(0.82-1.04)$ & 0.188 \\
\hline & No 23 & 84 & & & 209 & & \\
\hline \multirow[t]{2}{*}{ Neutropenia } & Yes 2 & 86 & $0.88(0.23-3.38)$ & 0.939 & 320 & $1.05(0.97-1.13)$ & 0.640 \\
\hline & No 28 & 85 & & & 175 & & \\
\hline \multirow[t]{2}{*}{ Anemia } & Yes 3 & 92 & $1.58(0.57-4.41)$ & 0.382 & 99 & $0.91(0.74-1.12)$ & 0.381 \\
\hline & No 27 & 86 & & & 195 & & \\
\hline \multirow[t]{2}{*}{ Thrombocytopenia } & Yes 1 & 85 & $0.88(0.14-5.74)$ & 0.895 & 548 & $1.21(0.63-2.31)$ & 0.569 \\
\hline & No 29 & 86 & & & 173 & & \\
\hline \multirow[t]{2}{*}{ Overall grade III/IV } & Yes 6 & 85 & $0.86(0.37-2.00)$ & 0.730 & 178 & $1.00(0.94-1.06)$ & 0.903 \\
\hline & No 24 & 87 & & & 187 & & \\
\hline
\end{tabular}

Bold values indicate that $p<0.05, O R$ odds ratio, $C I$ confidence interval

${ }^{\text {a }}$ OR is given per 10 percent increase in ratio $\mathrm{fT} 4$ or TSH 
Table 3 Univariate and multivariate logistic regression models of pCR and TSH and fT4

\begin{tabular}{|c|c|c|c|c|c|c|}
\hline & \multicolumn{3}{|c|}{ Univariate analysis } & \multicolumn{3}{|c|}{ Multivariate analysis } \\
\hline & OR & $95 \% \mathrm{CI}$ & $P$ value & OR & $95 \% \mathrm{CI}$ & $P$ value \\
\hline Clinical axillary lymph node: Pos versus Neg & 0.94 & $0.16-5.39$ & 0.942 & & & \\
\hline Clinical tumor size: $>5 \mathrm{~cm}$ versus $<5 \mathrm{~cm}$ & 0.61 & $0.10-3.82$ & 0.595 & & & \\
\hline $\mathrm{ER}+/ \mathrm{PR}+$ receptor: Pos versus Neg & 0.21 & $0.27-1.70$ & 0.154 & & & \\
\hline Total dose of CT & 1.01 & $0.88-1.16$ & 0.915 & & & \\
\hline fT4 & 0.78 & $0.43-1.42$ & 0.417 & 0.66 & $0.33-1.29$ & 0.223 \\
\hline TSH & 3.24 & $1.09-9.70$ & 0.035 & 19.7 & $0.77-504$ & 0.072 \\
\hline
\end{tabular}

Bold value indicates that $p<0.05, p C R$ pathological complete response, $E R$ estrogen receptor, $P R$ progesterone receptor, $O R$ odds ratio, $C I$ confidence interval in multivariate model adjusted for axillary lymph node status, tumor size and hormone receptor status

chemotherapy. In keeping with this inference, breast cancer survivors have a high cumulative incidence of overt primary hypothyroidism during long-term follow-up [24], and therefore, it would be interesting to measure fT4 and TSH concentrations during follow-up to evaluate whether or not the concentrations normalize after neoadjuvant chemotherapy. Alternatively, the increase of TSH we observed could also be explained in the context of recovery of "non thyroidal illness" (NTI), an adaptive response to (chemotherapy-induced) cellular damage. In critically ill patients, the hypothalamus-pituitary-thyroid axis down-regulates as an adaptation to adverse physical conditions [25]. Analogously, in another study of breast cancer patients treated with TEC or FEC, NTI-like plasma markers were observed one to 3 days after chemotherapy administration [19], suggesting that NTI may be a primary adaptive response to chemotherapy-induced cellular damage. In apparent contrast to our data, NTI is marked by a decline of TSH and secondary hypothyroidism, unlike the increase of TSH observed in our study. However, during recovery of critical illness and NTI, TSH levels tend to be increased [25]. Thus, the elevation of TSH we observed 3 weeks after chemotherapy administration, may in fact reflect the recovery of immediate adaptations of thyroid function (NTI) in response to treatment-induced cellular damage.

Whatever the cause of the chemotherapy-associated decline of fT4 we observed, a large reduction of fT4 concentrations in the course of 6 cycles TAC was associated with less (cumulative) side effects in terms of neuropathy, vomiting, and nausea of CTC grade II or higher. It is conceivable that lower levels of fT4 during chemotherapy, as an adaptive response, protect the body against tissue damage by down-regulating cellular metabolism. Interestingly, and potentially in analogy, low fT4 levels appear to protect people against the perils of old age, as low fT4 was associated with the longest lifespan in a population-based study of the elderly [26].

Influence of thyroid function on efficacy of cancer therapies has been described [27]. For example, in renal cancer patients treated with sunitinib, one-third of the patients experienced hypothyroidism as a side effect [27, 28], which had a positive impact on treatment outcome [29, 30]. Also, in a phase I/II study, propylthiouracil-induced chemical hypothyroidism was associated with a significantly longer median survival in glioma patients treated with high-dose tamoxifen [6]. Moreover, in patients with head and neck cancer, hypothyroidism due to radiotherapy tended to be associated with improved survival retrospectively [13]. However, we could not show that a low fT4 or a high TSH has a positive impact on chemotherapy efficacy.

It seems important to emphasize that the sample size of our study is small. Therefore, the results clearly need validation in a larger cohort which is planned in an ongoing trial in patients with early breast cancer (NCT02126449).

In conclusion, thyroid function declines during chemotherapy, and a large reduction of circulating fT4 is associated with less side effects of treatment. The results of our explorative study urge further evaluation of the impact of TH on cancer therapy.

Acknowledgments We are greatly indebted to the patients for participating in this (side) study, and we thank the LUMC Datacenter, department of Surgery, for trial coordination and data collection. This work was supported by grants from the Dutch Cancer Society (20104682), Amgen, Novartis and Sanofi Aventis.

Conflict of interest The authors declare that they have no conflict of interest.

Open Access This article is distributed under the terms of the Creative Commons Attribution Noncommercial License which permits any noncommercial use, distribution, and reproduction in any medium, provided the original author(s) and the source are credited.

\section{References}

1. Tosovic A, Bondeson AG, Bondeson L, Ericsson UB, Manjer J (2013) Triiodothyronine levels in relation to mortality from breast cancer and all causes: a population-based prospective cohort study. Eur J Endocrinol 168:483-490 
2. Tosovic A, Becker C, Bondeson AG et al (2012) Prospectively measured thyroid hormones and thyroid peroxidase antibodies in relation to breast cancer risk. Int J Cancer 131:2126-2133

3. Hellevik AI, Asvold BO, Bjoro T, Romundstad PR, Nilsen TI, Vatten LJ (2009) Thyroid function and cancer risk: a prospective population study. Cancer Epidemiol Biomarkers Prev 18: $570-574$

4. Moeller LC, Fuhrer D (2013) Thyroid hormone, thyroid hormone receptors, and cancer: a clinical perspective. Endocr Relat Cancer 20:R19-R29

5. Cristofanilli M, Yamamura Y, Kau SW et al (2005) Thyroid hormone and breast carcinoma. Primary hypothyroidism is associated with a reduced incidence of primary breast carcinoma. Cancer 103:1122-1128

6. Hercbergs AA, Goyal LK, Suh JH et al (2003) Propylthiouracilinduced chemical hypothyroidism with high-dose tamoxifen prolongs survival in recurrent high grade glioma: a phase I/II study. Anticancer Res 23:617-626

7. Eke KC, Turkmen YS, Temizel M et al (2013) Serum resistin and insulin-like growth factor-1 levels in patients with hypothyroidism and hyperthyroidism. J Thyr Res 2013:306750

8. Dinda S, Sanchez A, Moudgil V (2002) Estrogen-like effects of thyroid hormone on the regulation of tumor suppressor proteins, p53 and retinoblastoma, in breast cancer cells. Oncogene 21:761-768

9. Figueiredo NB, Cestari SH, Conde SJ et al (2014) Estrogenresponsive genes overlap with triiodothyronine-responsive genes in a breast carcinoma cell line. ScientificWorldJournal 2014: 969404

10. Hall LC, Salazar EP, Kane SR, Liu N (2008) Effects of thyroid hormones on human breast cancer cell proliferation. J Steroid Biochem Mol Biol 109:57-66

11. Vonderhaar BK, Greco AE (1982) Effect of thyroid status on development of spontaneous mammary tumors in primiparous C3H mice. Cancer Res 42:4553-4561

12. Pinto M, Soares P, Ribatti D (2011) Thyroid hormone as a regulator of tumor induced angiogenesis. Cancer Lett 301:119-126

13. Nelson M, Hercbergs A, Rybicki L, Strome M (2006) Association between development of hypothyroidism and improved survival in patients with head and neck cancer. Arch Otolaryngol Head Neck Surg 132:1041-1046

14. Ditsch N, Liebhardt S, Von KF et al (2010) Thyroid function in breast cancer patients. Anticancer Res 30:1713-1717

15. Cutuli B, Quentin P, Rodier JF, Barakat P, Grob JC (2000) Severe hypothyroidism after chemotherapy and locoregional irradiation for breast cancer. Radiother Oncol 57:103-105

16. Bruning P, Bonfrer J, De Jong-Bakker M, Nooyen W, Burgers M (1985) Primary hypothyroidism in breast cancer patients with irradiated supraclavicular lymph nodes. Br J Cancer 51:659-663
17. Joensuu H, Viikari J (1986) Thyroid function after postoperative radiation therapy in patients with breast cancer. Acta Radiol Oncol 25:167-170

18. Kailajarvi M, Ahokoski O, Virtanen A, Salminen E, Irjala K (2000) Alterations in laboratory test results during adjuvant breast cancer treatment. Clin Chem Lab Med 38:443-451

19. Huang J, Jin L, Ji G et al (2013) Implication from thyroid function decreasing during chemotherapy in breast cancer patients: chemosensitization role of triiodothyronine. BMC Cancer 13:334

20. Charehbili AV, van de Ven S, van d Smit VT et al (2014) Addition of zoledronic acid to neoadjuvant chemotherapy does not enhance tumor response in patients with HER2-negative stage II/III breast cancer: the NEOZOTAC trial (BOOG 2010-01). Ann Oncol 25:998-1004

21. Ogston KN, Miller ID, Payne S et al (2003) A new histological grading system to assess response of breast cancers to primary chemotherapy: prognostic significance and survival. Breast 12:320-327

22. Von MG, Untch M, Blohmer JU et al (2012) Definition and impact of pathologic complete response on prognosis after neoadjuvant chemotherapy in various intrinsic breast cancer subtypes. J Clin Oncol 30:1796-1804

23. Kuerer HM, Newman LA, Smith TL et al (1999) Clinical course of breast cancer patients with complete pathologic primary tumor and axillary lymph node response to doxorubicin-based neoadjuvant chemotherapy. J Clin Oncol 17:460-469

24. Khan NF, Mant D, Carpenter L, Forman D, Rose PW (2011) Long-term health outcomes in a British cohort of breast, colorectal and prostate cancer survivors: a database study. Br J Cancer 105(Suppl 1):S29-S37

25. Warner MH, Beckett GJ (2010) Mechanisms behind the nonthyroidal illness syndrome: an update. J Endocrinol 205:1-13

26. Gussekloo J, van Exel E, de Craen AJ, Meinders AE, Frolich M, Westendorp RG (2004) Thyroid status, disability and cognitive function, and survival in old age. JAMA 292:2591-2599

27. Hamnvik OP, Larsen PR, Marqusee E (2011) Thyroid dysfunction from antineoplastic agents. $J$ Natl Cancer Inst 103:1572-1587

28. Funakoshi T, Shimada YJ (2013) Risk of hypothyroidism in patients with cancer treated with sunitinib: a systematic review and meta-analysis. Acta Oncol 52:691-702

29. Sabatier R, Eymard JC, Walz J et al (2012) Could thyroid dysfunction influence outcome in sunitinib-treated metastatic renal cell carcinoma? Ann Oncol 23:714-721

30. Riesenbeck LM, Bierer S, Hoffmeister I et al (2011) Hypothyroidism correlates with a better prognosis in metastatic renal cancer patients treated with sorafenib or sunitinib. World J Urol 29:807-813 